\title{
Global health indicators: an overview
}

\author{
Charles Larson, Alec Mercer
}

ß See related article page 1203

T

The health of people living in developing countries around the world has improved considerably over the past 2 decades. For example, the total number of deaths annually among children less than 5 years of age has been halved over the past 40 years. ${ }^{1}$ Unfortunately, in most of sub-Saharan Africa and some parts of South Asia, these reductions in mortality have stagnated or even reversed over the past decade. ${ }^{2}$ At the same time, disease patterns and causes of death in less developed countries have changed markedly. The most obvious example of such change relates to HIV/AIDS, but others include the re-emergence of tuberculosis as a global health problem, the emergence of new infectious diseases, the increase in violence and the occurrence of chronic diseases formerly thought to be of concern only in developed countries, such as coronary artery disease and cerebrovascular accidents. ${ }^{3}$ Global monitoring of changes in the health of various populations requires the use of "tried and true" global health indicators. However, we also need indicators that are relevant to contemporary patterns of disease and their determinants.

This overview and the accompanying glossary of global health indicators (see the online appendix at www.cmaj.ca /cgi/content/full/171/10/1199/DC1) are intended to assist readers in understanding and interpreting summaries of global health statistics reported in professional journals and multinational publications, such as UNICEF's annual report, The State of the World's Children ${ }^{4}$ (see text box). Several global health indicators, such as infant mortality rate, are well known to health professionals working in both developed and developing countries. However, many others are not well understood and tend to bewilder readers or create "statistical overload" rather than accomplishing the intended purpose of improving understanding.

Global health indicators can be divided into those that directly measure health phenomena (e.g., diseases, deaths, use of services) and indirect measures (e.g., social development, education and poverty indicators); these are also referred to as proximal and distal indicators respectively. On the basis of population statistics describing levels of education attained and access to safe water and sanitation, it is possible to categorize a country fairly accurately as having a population with high, medium or low burden of disease. It is beyond the scope of this overview to address all potential indicators, and the glossary therefore focuses on direct measures of health, population and nutrition (or services related to these areas). The global health indicators used in developing countries for the most part address morbidity, mortality and important precursors of both. In contrast, in developed countries a large proportion of the key health indicators reflect lifestyles and individual behaviour, such as physical exercise, smoking, diet, or substance and alcohol abuse.

Because of cost constraints and limited logistic capacity, few developing countries are able to maintain death, birth or disease registries. Data generated by health care institutions are more readily available and more frequently used, but must be viewed with caution because they are not representative of the general population. In developing countries, a biased minority of the population (typically urban, wealthy and better educated) use modern medical services. Furthermore, for several presenting health problems, only patients exhibiting the most severe end of the spectrum ever reach a health care facility. Fortunately, alternative, technologically appropriate, affordable data collection methods have been adopted in many developing countries, including national demographic and health surveys, one-off ecologic or household surveys, multiple-indicator cluster surveys, verbal autopsies and demographic surveillance systems within selected populations. A combination of data from these sources is then used to assess the health status of the nation as a whole or regionally.

There are far more health indicators to choose from than any health information system can reasonably include. In developing countries, because of severely restricted resources and capacities, there is a need to select a limited set of indicators that are clearly applicable to monitoring, decision-making and health policy, and to measure them well. What characteristics should guide the selection of a global health indicator? Few indicators meet all of the following criteria, but some do much better than others.

- Definition. The indicator must be well defined, and the definition must be uniformly applied internationally.

- Validity. The indicator must be valid (it must actually measure what it is supposed to measure), reliable (replicable and consistent between settings) and readily interpretable.

- Feasibility. The gathering of the required information must be technologically feasible and affordable and must not overburden the system.

- Utility. The indicator should provide information that is useful to decision-makers and can be acted upon at various levels (local, national and international).

The validity of published health statistics from developing countries is variable and extremely difficult to assess. Validity can be threatened in several ways. For instance, it is ad- 
versely affected by variations between countries in definitions of health and illness states, by the choice of easy-toreach but usually unrepresentative populations for surveys, and by the underestimation or overestimation of denominators. Denominators tend to be based on the most recent census and the application of crude death and birth estimates. However, such data are often out of date and are vulnerable to manipulation. Numerators, such as the occurrence of an illness or health behaviour, are also subject to multiple sources of error. They depend on a respondent's capacity to understand the question and to recall an event or exposure, as well as a willingness to report what he or she recalls.

\section{Web sites presenting data, publications and other information relevant to global health and global health indicators}

\section{World Health Organization (WHO) Statistical Information System}

www3.who.int/whosis/menu.cfm

A guide to health and health-related epidemiological and statistical information available from the WHO, including information from WHO technical programs

\section{Global Health Council}

www.globalhealth.org

Useful updates and excellent links to other resources

United Nations Children's Fund (UNICEF)

www.unicef.org

Descriptions of international initiatives and links to UNICEF publications, including the annual State of the World's Children report

\section{US Centers for Disease Control and Prevention, National Center for Environmental Health}

www.cdc.gov/nceh/indicators/default.htm

Information on the Environmental Public Health Indicators Project, including an extensive list of health indicators and their definitions

\section{United Nations Development Programme}

www.undp.org

Includes annual Human Development Report, covering all human resource sectors and providing data and statistics derived from official statistical systems organized by national governments

\section{Save the Children}

www.savethechildren.org/publications

Valuable resource for pediatric mortality and morbidity data, including State of the World's Newborns (2001)

\section{Statistics Canada}

www.statcan.ca

Annual reporting of vital statistics and other health indicators for Canada and by province
How can the validity and utility of global health indicators be improved? Countries and health care systems with restricted resources can consider several options, including the following:

- measurement of a smaller number of indicators, specifically those with direct relevance to decision-making and high-priority health issues

- use of more efficient sampling frames and procedures

- continued refinement and validation of verbal autopsies

- application of standard, internationally accepted definitions.

Despite the acknowledged limitations of many health indicators and the reservations one might have about the accuracy of health statistics derived in developing countries, they nonetheless do provide useful estimates of a population's state of health. Although the absolute estimates may lack accuracy, they constitute a valuable "yardstick" by which to monitor change within a population over time. Comparison between countries is also possible, but is more susceptible to information and selection bias. Global health indicators highlight the tremendous disparities in health status within a given population or between developing and developed countries. Withincountry and subpopulation comparisons are becoming more common as health care professionals in the developing world become more aware of and more concerned about the performance of health care systems and issues of equity in relation to gender, socioeconomic status, ethnicity and geography.

In summary, it is reasonable to question the accuracy of global health indicators reported from developing countries. However, intermediate technologies applied by developing countries, such as national demographic and health surveys, do produce acceptable estimates of these indicators and can provide valuable information for policy-makers, allowing them to monitor equitable progress toward healthier populations.

From the Department of Pediatrics and the Joint Department of Epidemiology, Biostatistics and Occupational Health, McGill University, Montréal, Que. (Larson); and the Centre for Health and Population Research (ICDDR,B), Dhaka, Bangladesh (Larson, Mercer)

Competing interests: None declared.

Contributors: Charles Larson was the lead author and primarily responsible for the preparation of the manuscript and the glossary. Alec Mercer made important revisions to the initial draft and provided original intellectual input to the final version.

\section{References}

1. Black RE, Morris SS, Bryce J. Where and why are 10 million children dying every year? Lancet 2003;361:2226-34.

2. Progress for children. A child survival report card. Vol. 1. New York: UNICEF; 2004. Available: www.unicef.org/media/files/29652L01Eng.pdf (accessed 2004 Oct 12).

3. Yusuf S, Reddy S, Ounpuu S, Anand S. Global burden of cardiovascular diseases: Part I: general considerations, the epidemiologic transition, risk factors, and impact of urbanization. Circulation 2001;104:2746-53.

4. The state of the world's children 2004. New York: UNICEF; 2004. Available: www.unicef.org/sowc04 (accessed 2004 Oct 7).

Correspondence to: Dr. Charles Larson, Centre for Health and Population Research (ICDDR,B), GPO Box 128, Dhaka 1000, Bangladesh; fax 514 398-4503; clarson@icddrb.org 